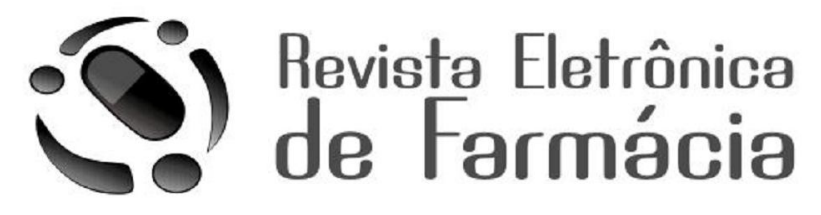

REF - ISSN 1808-0804 Vol. VIII (1), 22 - 28, 2011

\title{
AVALIAÇÃo dOS EFEITOS do EXTRATO METABÓLICO DE Stryphnodendrom adstringes (Mart) Coville POR GAVAGeM NO CICLO ESTRAL DE RATAS
}

\author{
EVALUATION OF EFFECTS OF METABOLIC EXTRACT OF Stryphnodendrom tightens (Mart) Coville \\ FOR GAVAGEM IN THE CICLO ESTRAL OF FEMALE RATS
}

\section{Suize Silva Oliveira ${ }^{1, *} \&$ Maria Luzinete Alves Vanzeler ${ }^{2}$}

\author{
${ }^{1}$ Medicina Veterinária, MSc. Saúde Coletiva. Endereço: Rua Professora Silvia Curvo 560 - Cidade Alta \\ 78030-440. \\ ${ }^{2}$ Professora Dr. Departamento de Ciências Básicas em Saúde (DCBS) da Faculdade de Ciências \\ Médicas (FCM) da Universidade Federal de Mato Grosso (UFMT), CEP 78060-900 Cuiabá MT, \\ telefones: 55XX65-36158865. \\ * E-mail do autor Correspondente: suizeoli@bol.com.br
}

Recebido em 01/ 09/ 2010, Aceito em 02/ 02/ 2011

RESUMO: O Stryphonodendron adstringens (Mat.) Coville, também conhecido como barbatimão, tem sido utilizado na medicina popular para o tratamento de diarréia e ulceras de estomago para enterorragias, metrorragias, e hemorragias uterinas durante a gravidez, entre outras indicações. O objetivo deste trabalho foi avaliar os efeitos do extrato metabólico de Stryphnodendrom adstringes (Mart) Coville (EMSa) (Barbatimão), no ciclo estral de ratas. Para este procedimento, foram utilizadas 80 ratas de aproximadamente 2 meses e meio de idade, virgens, sadias e ciclando normalmente por três ciclos consecutivos. As ratas foram randomicamente divididas em 4 grupos de 20 animais e tratadas por 15 dias com EMSa 200,400, $800 \mathrm{mg} \cdot \mathrm{kg}^{-1}$ ou água destilada, por gavagem orogástrica em volume constante $\left(0,1 \mathrm{~mL} \cdot \mathrm{kg}^{-1}\right.$ de peso corporal). O ciclo estral foi acompanhado através de observações microscópicas diárias do esfregaço vaginal conforme Ham (1967). Na investigação da toxidez do EMSa neste trabalho, foi possível verificar que a utilização deste extrato metabólico não alterou o ciclo estral das ratas, sendo que $100 \%$ das ratas controle e tratadas com EMSa nas doses de 200, 400 e 800 mg. $\mathrm{kg}^{-1}$ ciclaram normalmente durante os quinze dias de tratamento; portanto, observou-se que o extrato não apresenta efeito anticonceptivo.

Palavras chave: Barbatimão, esfregaço vaginal, toxicidade.

ABSTRACT: The Stryphonodendron adstringens (Matt) Coville, also known as "barbatimão", has been used in folk medicine to treat diarrhea, stomach ulcers, rectal bleeding, metrorrhagy and uterine bleeding during pregnancy, among other indications. The aim of this study was to evaluate the metabolic effects of the extract Stryphnodendron astringe (Mart) Coville (EMSA) (barbatimão) on estrous cycle of female rats. For this procedure, 80 healthy virgin $21 / 2$-month-old rats were used, having normally cycled for three consecutive cycles. The rats were randomly divided into four groups of 20 animals and treated for 15 days with EMSA 200, 400, $800 \mathrm{mg} \cdot \mathrm{kg}^{-1}$ or distilled water by orogastric gavage in a constant volume $\left(0.1 \mathrm{~mL} \cdot \mathrm{kg}^{-1}\right.$ body weight). The estrous cycle was monitored by daily microscopic observations of vaginal smears according to Ham (1967). In investigating the toxicity of EMSA in this study, we found that the use of this extract did not alter the metabolic 
estrous cycle of female rats, as $100 \%$ of control rats and rats treated with EMSa in doses of 200, 400 and $800 \mathrm{mg} \cdot \mathrm{kg}^{-1}$, normally cycled during the fifteen days of treatment; thus, it was observed that the extract does not present any contraceptive effect.

Keywords: barbatimão, vaginal smear, toxicity.

\section{NTRODUÇÃO}

Atualmente a grande procura por terapias alternativas tem aumentado 0 interesse pelas plantas utilizadas na medicina popular. Segundo Gossell-Williams et al., este interesse se deve ao alto custo dos medicamentos alopáticos e à busca da população por tratamentos menos agressivos ao organismo humano. Os medicamentos fitoterápicos são preparações farmacêuticas (extratos, tinturas, pomadas e cápsulas) de ervas medicinais, obtidos a partir de uma ou mais plantas e utilizados para o tratamento de várias doenças. Inúmeras são as vantagens para o uso terapêutico, como o baixo custo e a grande disponibilidade para a população de baixa renda.

O Stryphonodendron adstringens (Mat.) Coville, também conhecido como barbatimão, apresenta sua área de dispersão limitada ao continente sul-americano. Caracteriza-se como planta neotropical, apresentando como limite norte a Costa Rica, na America Central, com registro de uma única espécie (S. poliphyllum) e, como limite sul, o estado do Paraná, no Brasil com as espécies $S$. obovatum e S. adstringens. Esta planta tem sido utilizada na medicina popular para o tratamento de diarréia e ulceras de estomago, para enterroragias, metrorragias, antileucorreica hemostática ou paralisante das hemoptises e hemorragias uterinas durante a gravidez, combate as ulceras, escorbúticas, hérnias, empinges, cicatrização de feridas, entre outras indicações. Possui como constituintes químicos alcalóides, flavonóides, terpenos, estilbenos, esteróides, inibidores de proteases (como a tripsina) e taninos. Taninos são os componentes majoritários do barbatimão, sendo que estes compostos têm sido associados com efeitos antimicrobianos. Os taninos vegetais são classificados em dois grupos: hidrolisáveis e condensados. Os taninos hidrolisáveis dividem-se em galotaninos e elagitaninos, que produzem, respectivamente, ácido gálico e ácido elágico após hidrólise. Estes taninos se apresentam em menor quantidade em madeiras do que os taninos condensados. No processo de cura de feridas, queimaduras e inflamações os taninos formam uma camada protetora sobre a mucosa ou tecido lesado, através do complexo tanino-proteína e/ou polissacarídeos. $\mathrm{Na}$ úlcera gástrica, provavelmente ocorre um processo similar, onde a camada protege a mucosa gástrica.

Existem três propriedades gerais dos taninos que são responsáveis pela maior parte das atividades farmacológicas destas substâncias: a formação de complexos com íons metálicos (ferro, alumínio, cálcio, cobre, etc. ), a atividade antioxidante e seqüestradora de radicais livres e a habilidade de formar complexos com outras moléculas tais como proteínas e polissacarídeos. Estudos anteriores mostraram ação anti-edematogênica de solução de barbatimão a 1\%, atividade antibacteriana para Staphylococcus aureus e 
Pseudômonas aeruginosa e hipotensora dos extratos acêtonicos e semipurificados da casca de barbatimão. Orlando observou atividade antimicrobiana in vitro do extrato hidroalcoólico bruto da casca do barbatimão para Enterococcus faecalis, Kocuria rhizophila, Escherichia coli, Neisseria gonorrhoeae, Pseudômonas aeruginosa.

Observando o grande número de utilizações do barbatimão na medicina popular, verificou-se a necessidade de esclarecer se existe riscos de distúrbio hormonais femininos no uso do extrato metabólico de Stryphnodendrom adstringes (Mart) Coville (EMSa) (Barbatimão), para tanto, este trabalho teve como objetivo avaliar os efeitos do EMSa, no ciclo estral de ratas.

\section{MATERI AL E METODOS}

Este estudo foi aprovado pelo Comitê de Ética da Universidade Federal de Mato Grosso (Processo número 23108014845/8-2), e os estudos foram realizados de acordo com as regras do Código de Ética Profissional do Médico Veterinário e do Colégio Brasileiro de Experimentação Animal (COBEA).

As cascas do Stryphnodendron adstringens foram coletadas nos arredores da rodovia Vicente Bezzera Neto, km 80 na comunidade Poço Fundo localizada em Chapada dos Guimarães-MT e Município de Santo Antonio do Leverger, no km 60 da rodovia 364. As cacas foram secas ao ar e posteriormente trituradas em moinho de facas TE 625 (TECNAL). O material resultante (pó) foi separado em porções de $250 \mathrm{~g}$ em frascos de vidro. Em cada frasco de vidro foi adicionado 2,5 L de solução hidroalcoólica (70\%) e macerado durante 7 dias, com homogeneização diária e armazenados em local adequado para reduzir a incidência de luz sobre o material. $O$ extrato foi filtrado e levado a uma temperatura de $42 \pm 1 \stackrel{\circ}{ } \mathrm{C}$, para a evaporação do solvente em um evaporador rotatório a bomba a vácuo Tecnal TE-058, sob pressão de $625 \mathrm{mmHg}$. O extrato bruto obtido foi retirado dos frascos e depositados em cápsulas de porcelana, deixando-os em estufa a $45 \pm 2$ 으 para secagem, obtendo-se o assim o extrato metabólico bruto de barbatimão (EMSa). O EMSa foi armazenado em frasco âmbar e mantido e vedado dentro de geladeira a $4 \pm 2^{\circ} \mathrm{C}$.

O experimento foi realizado no Laboratório de Psicofarmacologia da Faculdade de Medicina da Universidade Federal de Mato Grosso - UFMT. Para este procedimento, foram utilizadas 80 ratas de aproximadamente 2 meses e meio de idade, virgens, sadias e ciclando normalmente por três ciclos consecutivos. Todas provenientes do cruzamento sucessivo no Biotério Central da Universidade Federal de Mato Grosso. Estes animais foram submetidos a 18 horas de jejum alimentar, sem restrição a água. E posteriormente, as ratas foram randomicamente divididas em 4 grupos de 20 animais e tratadas por 15 dias com EMSa 200, 400, $800 \mathrm{mg} \cdot \mathrm{kg}^{-1}$ ou água destilada, por gavagem orogástrica em volume constante $\left(0,1 \mathrm{~mL} \cdot \mathrm{kg}^{-1}\right.$ de peso corporal).

O extrato sempre era preparado minutos antes de ocorrer os tratamentos. Pesava-se o pó resultante da extração em balança analítica e adicionava-se água destilada, homogeneizando bem em um Becker com o auxilio de uma espátula de porcelana.

O ciclo estral foi acompanhado através de observações microscópicas diárias do esfregaço vaginal conforme Ham. Os 
esfregaços foram feitos sempre pela manhã das 8 às 9 horas, através da aplicação de 0,5 $\mathrm{mL}$ de solução de $\mathrm{NaCl} 0,9 \%$ na vagina, com uma pipeta de plástico de $1 \mathrm{~mL}$, seguido por aspiração do lavado. O material pipetado foi depositado sobre uma lâmina e levado ao microscópio ótico monocolor e observadas às fases do ciclo estral, segundo Hankness e Wagner: a) diestro - presença de muitos leucócitos e/ou muco; b) proestro - presença de células epiteliais nucleadas; c) início do estro - muitas células epiteliais cornificadas presentes e algumas células nucleadas; d) final do estro - muitas células epiteliais cornificadas juntas; e) meta-estro - presença de células cornificadas e leucócitos em grande quantidade (Figura 1).

Considerou-se normal à duração de 4 a 5 dias de ciclo estral, com o período de cio de aproximadamente 12 horas e de ocorrência noturna, conforme Hankness e Wagner.

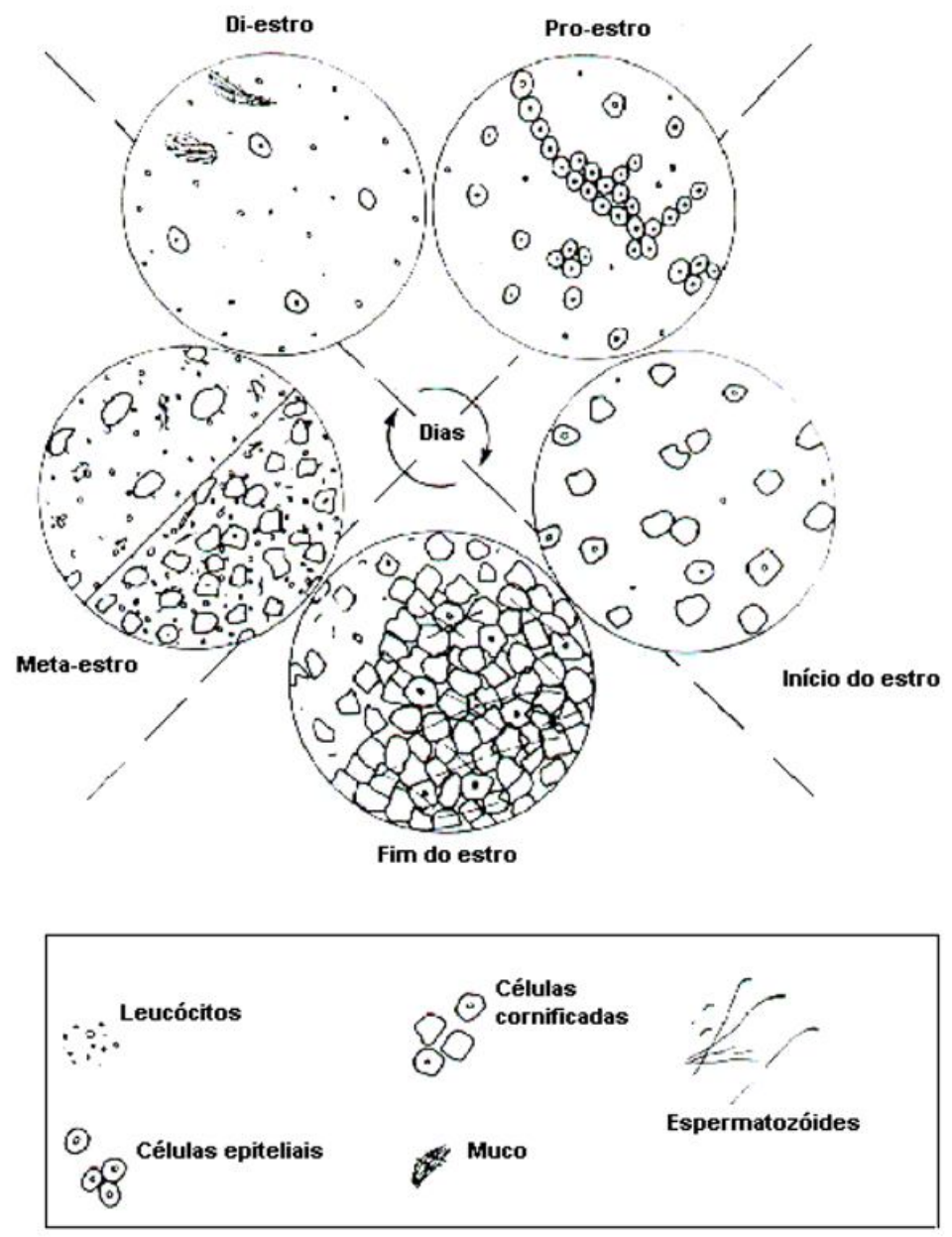

Figura 1. Interpretação do lavado vaginal

Fonte: BARROW.

\section{RESULTADOS E DISCUSSÃO}

A avaliação dos efeitos do extrato metabólico bruto de Stryphonodendron adstringens (Mart.) Coville no ciclo estral de ratas vai de encontro à tendência atual de se desenvolver e testar medicamentos produzidos a partir de plantas naturais. 
A atividade estrogênica das plantas foi primeiramente demonstrada em 1926, e em meados da década de 70 já se tinha demonstrado que centenas de plantas exibiam atividade estrogênica. Os fitoestrogênios assumiriam importância biológica e econômica nos anos 40 , com a diminuição da fertilidade induzida em ovelhas pela ingestão de trevos de pastagens, na Austrália, na chamada "doença do trevo".

Em relação aos efeitos do extrato EMSa não se observou qualquer alteração comportamental das ratas. Verificou-se que a utilização do extrato metabólico não alterou o ciclo estral das ratas, onde, $100 \%$ das ratas controle e tratadas com EMSa nas doses de 200, 400 e 800 mg. $\mathrm{kg}^{-1}$, ciclaram normalmente durante todo o tratamento.

Grande parte do conhecimento sobre o controle do ciclo ovariano de vários mamíferos, é baseado em estudos sobe o ciclo estral de ratas.

O envolvimento do Sistema Nervoso Central em regular a secreção da hipófise anterior, estimulando a expressão de comportamentos reprodutivos e monitorando as aferências sensoriais do meio ambiente faz do ciclo um processo neuroendócrino.

Sabe-se que a elevação plasmática do hormônio Luteinizante no proestro é fundamental para os eventos que culminarão com a ovulação.

Estudos realizados por diversos pesquisadores, dentre estes cita-se Vitral et al., demonstraram que S. obovatum Benth e S. pollyphyllum M. apresentaram efeitos abortivos e de má formação da prole.

Neste modelo experimental, sugere-se que não houve um distúrbio no eixo hipotálamo-hipófise-gônadas, não havendo interferência no perfil hormonal em ratas tratadas e, portanto, no ciclo estral.

Condição que não descarta estudos futuros com EMSa utilizando-se coelhos, espécie que possui elevada sensibilidade a agentes teratogênicos para o homem, e possuem características anatômicas e embriológicas comuns as ratas e camundongos.

Tabela 01. Influência da administração de EMSa ou veículo por quinze dias no ciclo estral de ratas

\begin{tabular}{cccc}
\hline \multirow{2}{*}{$\begin{array}{c}\text { Doses de } \\
\text { EMSa mg.kg }\end{array}$} & N & $\begin{array}{c}\text { Ciclo Estral } \\
\text { ciclaram (\%) }\end{array}$ & $\begin{array}{c}\text { Duração do ciclo em dias } \\
\text { (Média } \pm \text { E.P.) }\end{array}$ \\
\hline 0 & 20 & 100 & $4,3 \pm 0,1 \mathrm{a}$ \\
200 & 20 & 100 & $4,4 \pm 0,1 \mathrm{a}$ \\
400 & 20 & 100 & $4,0 \pm 0,3 \mathrm{a}$ \\
800 & 20 & 100 & $3,2 \pm 0,2 \mathrm{a}$ \\
\hline
\end{tabular}

Letras iguais na mesma coluna não diferem entre si pelo teste de Dunnett $(p<0,01 \%)$. 
A partir deste trabalho, foi possível concluir que as diferentes doses de EMSa quando comparado ao grupo controle, não levou a alteração do ciclo estral das ratas,

\section{AGRADECIMENTOS}

A todos que contribuíram para a realização deste trabalho. submetidas a investigação por 15 dias.

\section{REFERENCIA BIBLI OGRAFICA}

Almeida SP, Proença C B, Sano SM, Ribeiro JF. Cerrado, espécies vegetais úteis. Embrapa, Planaltina - DF, $1998.464 p$.

Audi EA, Toledo CEM, Santos FS, Bellanda PR, Prado, WA, Nakamura TU et al. Biological activity and quality control of extract and stem bark from Stryphnodendron adstringens. Acta Fam Bonaerense 2004; 23:328-33.

Barrow AS. Technical procedures in reproduction toxicology. London, Royal Society of Medicine services limited for Laboratory Animals Ltd., 1990.p 1-55.

Berg ME. van den. Plantas Medicinais na Amazônia: Contribuição ao seu conhecimento sistemático. Belém: Museu Paraense Emílio Goeldi. 1993.p 207.

Calixto JB. Efficacy, safety, quality control, marketing and regulatory guidelines for herbal medicines (phytoterapeutic agents). Braz J Med Biol Res 2000;33: 79-89.

Clapauch R, Meirelles RMR, Julião Masg, Loureiro CKC, Giarodoli PB, Pinheiro AS, Harrigan AR, Sprintzer PM, Pardini DP, Weiss RV, Athaye A, Russo L A \& Povoa LC. Fitoestrogênio, posicionamento do departamento de endocrinologia feminina da Sociedade Brasileira de Endocrinologia e Metabologia (SBEM). Arquivo Brasileiro de Endocrinologia e Metabologia, 2002.

Coimbra, R. Manual de Fitoterapia. Belém: CEJUP, 1994.335p.

Coutinho H, Pinto DS, Ribeiro JE, Friedman H. Ação antiedematosa do Stryphnodendron barbadetiman (Barbatimão) a $1 \%$ em comparação a clorexidina a $0,12 \%$. Rev odonto cienc 2004; 19:201-6. 
Freeman M.E. The ovarian cycle of the rat. In: Knobil, E. \& Neill, J. (eds). The Physiology of Reproduction. New York: Raven Press, 1994.

Gossell-Williams M, Simon OR, West ME. The past and present use of plants for medicines. West Indian Med J 2006;55:217-8.

Ham AW. Histologia. Rio de Janeiro: Ed Guanabara Koogan S.A.; 1967.

Hankness JE, Wagner JE. Biologia e Clínica de Coelhos e Roedores. São Paulo: Roca, 3o edição, $1993.238 \mathrm{p}$.

Haslam, E. Natural polyphenols (vegetable tannins) as drugs and medicines: possible modes of action. J Nat Prod 1996; 59: 205-15.

Mello, JC. Plantas em Destaque: Barbatimão (Córtex). Rev. Racine. 1998;46:42-3.

Occhioni E ML. - Considerações taxonomicas no gênero Stryphnodendron adstringens.(Leguminosae - Mimosoidae) e distribuição geográfica das espécies. Acta Bot. J. Pharmacol., 1963.21: 127-136.

Orlando SC. Avaliação da atividade antimicrobiana do extrato hidroalcoólico bruto da casca de Stryphnodendron adstringens (Martius) Coville (Barbatimão) [dissertação]. Franca (SP): Universidade de Franca; 2005.

Queiroz CRAA, Morais SAL, Nascimento EA. Caracterização dos taninos da aroeira preta (Myracrodruon urundeuva). Rev Árvore, 2002;26:493-7.

Santos SC, Costa WF, Batista F, Santos LR, Ferri PH, Ferreira HD. et al. Seasonal variation in the content of tannins in barks of barbatimão species. Rev bras farmacogn 2006; 16: 552-6.

Silva HVC. Ação da Stryphnodendron barbatiman sobre a cicatrização: estudo experimental em ratos. HB cient; jan.-abr.1996. 3(1):77-9.

Vitral GSF, Peters VM, Guerra MO. Mecanismos da ação embriotóxica do barbatimão (Stryphnodendron polyphyllum M.). 1987. Reprodução; (3):222-6. 